\title{
Erratum
}

\section{Erratum. SRY-Box Containing Gene 4 Promotes Liver Steatosis by Upregulation of SREBP-1C. Diabetes} 2018;67:2227-2238

Yang Jiao, Jiejie Zhao, Zhijian Zhang, Min Li, Xi Yu, Yanying Yang, Jie Liu, Shengjie Liao, Duanzhuo Li, Yuxing Wang, Die Zhang, Yulu Chen, Guojun Shi, Bin Liu, Yan Lu, and Xiaoying Li

https://doi.org/10.2337/db19-er01b

In the article listed above, the top two panels in Fig. $2 D$ with a magnification of $\times 100$ were inadvertently misused and have been replaced with Oil Red O staining with a magnification of $\times 200$. The authors apologize for this error.

The online version of the article (https://doi.org/10.2337/db18-0184) has been updated to correct this error. 